\title{
Gut microbiome dysbiosis in metabolic disorders: implications for probiotics as prospective investigational new drugs
}

\author{
Praveen P. Balgir, Baljinder Kaur, Tejinder Kaur \\ Department of Biotechnology, Punjabi University, Patiala, India
}

\begin{abstract}
Gut microbiome has been analysed using metagenomics approach which combines the power of genomics, bioinformatics, and systems biology, providing new ways to access the microbial world. Gut microbiome evolves from the pre-birth exposure onwards in a child, as a result of its interaction with its environment; and is apparently influenced by various environmental factors all through the life, most important being diet and its composition. Metagenomic analysis of gut microbial communities of a malnourished and a healthy child revealed an abundance of enteric pathogens leading to intestinal inflammation and nutrient malabsorption. The change in diet from vegetarian to non-vegetarian also influences the composition and dynamics of the intestinal microbes.

Gut microbiota have been observed to vary with age due to physiological changes in the gastrointestinal tract, modifications in lifestyle, nutritional behavior and weakened functionality of the host immune system. Studies in apparently healthy individuals and patients suffering from intestinal or metabolic disorders revealed differential microbiome compositions, proving that an imbalance of gut microflora leads to dysbiosis which is the major cause of metabolic disorders such as irritable bowel syndrome (IBS), obesity, diarrhea, etc. The investigational studies are trying to assess the relationship between intestinal microbiota dysbiosis and various metabolic diseases. Nutritional interventions in the form of probiotics and prebiotics as investigational new drugs (INDs) are being assessed for correcting dysbiosis in disease states. The current paper focuses on the recent knowledge and databases for the same with pointers to future investigations as the need of the hour studies.
\end{abstract}

Keywords: Dysbiosis, malnutrition, metagenome, microbiome, probiotic

\section{INTRODUCTION}

Microbes have colonized all niches in human body and have attracted the attention of the scientific community to understand the role played by these commensal communities in human health. Throughout the world a number of major projects to delineate the

Corresponding Author: Prof. Praveen P. Balgir

E-mail: balgirbt@live.com

Received:16-07-2015

Accepted: 24-09-2015

How to cite this article: Balgir PP, Kaur B, Kaur T. Gut microbiome dysbiosis in metabolic disorders: Implications for probiotics as prospective investigational new drugs. J Gastrointest Infect, 2015; 5: 5-12. interactions of microbes with human host and also with other microbial neighbors present on human body are presently in progress. Gastrointestinal tract is the largest organ in human body providing these microbial colonizers with a number of eco-niches within this single continuum. The metabolic activities of these microbes are crucial to physical and mental well-being of the host. ${ }^{[1]}$

\section{Metagenomics for microbiome analysis}

A number of methodologies have been used for microbiome analysis. Metabolomic studies using physiological profiling by BIOLOG and MALDI-TOF MS studies have been used to classify complex microbial 
communities. ${ }^{[1]}$ Metagenome analysis also reveals microbiome composition. A number of genome based methodologies like multi locus analysis using Random Amplified Polymorphic DNA (RAPD), Polymerase chain Reaction Based Denaturing Gradient Gel Electrophoresis (PCR -DGGE), PCR based Multi Locus Sequence Typing (PCR-MLST), Single Strand Confirmational Polymorphism (SSCP) and quantitative multiplex (qPCR) have been used for microbiome analysis. The most commonly used microbiome analysis tool is based on 16s ribosomal RNA sequencing. Other recent advances in culture-independent techniques for characterization of microbial community that have led to major progress in understanding the complexity of the human intestinal ecosystem ${ }^{[2,3,4]}$ include highthroughput amplicon pyrosequencing ${ }^{[5]}$ and total shotgun sequencing of the microbial metagenome, ${ }^{[6]}$ but the cost for these techniques is currently prohibitive for routine application to a large number of samples.

Metagenomic studies of the human intestinal microbiome have revealed that human gut carries, on an average, about 540,000 microbial genes, representing the dominant microbes in this ecosystem. Approximately $55 \%$ of these genes constitute the core metagenome (i.e., are genes shared among at least $50 \%$ of individuals), while many other genes appear to be unique and/ or present in less than $20 \%$ of individuals. A disturbed balance of beneficial and detrimental bacteria (dysbiosis) could promote intestinal inflammation and other metabolic disorders. ${ }^{[7,8]}$

\section{Microbiome growth and development}

Humans are born with a sterile gastrointestinal tract that is successively colonized with microbial populations until adult-like communities stabilize. ${ }^{[9]}$ This process has been described as chaotic, owing to a lack of commonly shared temporal patterns of colonization in infants and an incomplete understanding about the events that shape patterns of colonization. ${ }^{[2,9]}$ Current theory in community ecology proposes three processes that drive community assembly. These are (i) deterministic niche-related, (ii) historic, and (iii) neutral. ${ }^{[10,1]}$ All three types of processes likely combine to shape the assembly of the gut microbiota, but the ecological features of the human gut microbiota suggest differences in their relative importance.
The initial infant gut microbiota, acquired during passage through birth canal at parturition or later during breastfeeding is simple in composition and is usually dominated by bifidobacteria, and through a series of successions and replacements, it develops into the more complex adult pattern. ${ }^{[2,12]}$

\section{Microbiome maintenance}

The lifestyles of individuals affect the microbiome evolution and content of gut microbes. Due to improved hygiene and nutrition, the western human diet contains very less variety of bacteria as compared to preindustrialized diets. The lack of microbial variation is due to use of processed and sterile food ingredients, artificial sweeteners and preservatives, rather than fresh fruits and vegetables that are food items containing important microbes of anti-inflammatory nature. ${ }^{[13,14]}$

Changes in dietary consumption patterns affect many aspects of human biology. David et al ${ }^{[14]}$ reported alterations in microbial community structure after the consumption of diets composed entirely of either animal or plant products. It was observed that consumption of a completely vegetarian diet lead to a greater abundance of Prevotella community in gut, whereas Bacteroides were found to decrease. On the contrary, gut of a nonvegetarian; had reversed ratios of these two microbial communities.

The metabolite sensors on immune cells of gut sense the dietary changes and respond to the determinants of nutritional status such as vitamins etc. The changing diet also influences the composition and dynamics of the intestinal microbes. This composition of host gut microbiome is known to play an extremely important role in absorption of nutrients from food and response to calorie content. Thus similar calorific content of food in different individuals, may also lead to malnutrition. On the other hand even enteric infections result in malabsorption of nutrients due to intestinal inflammation and contribute to malnutrition in large proportions of children in developing countries. ${ }^{[15]}$

Gupta et $a l^{[16]}$ examined the variations in gut microbiota of a malnourished child from a typical urban slum in Kolkata and an apparently healthy child. Results of this investigation revealed dysbiosis in gut microflora in case of malnourished child when compared with gut microbial composition of the healthy child. This 
imbalance in microbial community composition was proposed to lead to inflammation and malabsorption in the malnourished subjects. To completely understand the role of gut microbiota in malnutrition amongst millions of children across the globe, models from different socioeconomic backgrounds, geographically distinct locations and diverse age groups need to be investigated.

\section{Dysbiosis}

Proper functioning and homeostasis of our intestine relies on an intimate and symbiotic relationship between our mucosal surface, the microbiota and its metabolic by-products. Homeostasis is characterized by the diverse microbiota producing a variety of metabolites such as short chain fatty acids (SCFAs), biogenic amines (such as histamine) or other amino-acid-derived metabolites such as serotonin or gamma-aminobutyric acid, coupled to a lack of pathology associated with specific T-cell subsets. ${ }^{[17]}$ Imbalance in the intestinal microbiota referred to as dysbiosis, is increasingly linked to intestinal diseases (Fig.1). Disturbances in the intestinal microbiota caused by infections and antibiotics have profound effect on the microbiota's composition and function and have been observed to predispose the individual to antibiotic-associated diarrhea.
Dysbiosis has been associated with illnesses, such as inflammatory bowel disease (IBD) ${ }^{[18,19,20]}$ chronic fatigue syndrome ${ }^{[21]}$ obesity, ${ }^{[2,23]}$ cancer, ${ }^{[24,25]}$ bacterial vaginosi ${ }^{[26]}$ and colitis. $^{[27]}$ The first line treatment for severe Clostridium difficile infections includes metronidazole or vancomycin although in $15-35 \%$ of these cases, a recurrence (relapse or re-infection) follows the cessation of antibiotic therapy. Fecal microbiota transplantation (FMT) has been mainly used as an alternative treatment for patients with persistent, recurrent $C$. difficile infection and involves the restoration of the intestinal microbiota through installation or engraftment of homogenized fecal suspension from a healthy donor. FMT has also been utilized in the treatment of diseases associated with intestinal dysbiosis such as IBD manifested as Crohn's disease and ulcerative colitis.

Changes in the adult gut microbiota have also been linked to other undesirable health conditions such as obesity. ${ }^{[28]}$ The link between obesity and the microbiota is likely to be more sophisticated than the simple phylum-level Bacteroidetes: Firmicutes ratio initially identified ${ }^{[29]}$ and it is likely to involve microbiota-diet interaction as well. ${ }^{[30,31]}$ Faecalibacterium prausnitzii, has been found to be depleted in IBD patient ${ }^{[7,3]}$ along with anti-inflammatory activity in vitro and in mouse models, ${ }^{[8]}$ whereas adherent Escherichia coli cells have been found to increase with IBD. ${ }^{[32,33]}$

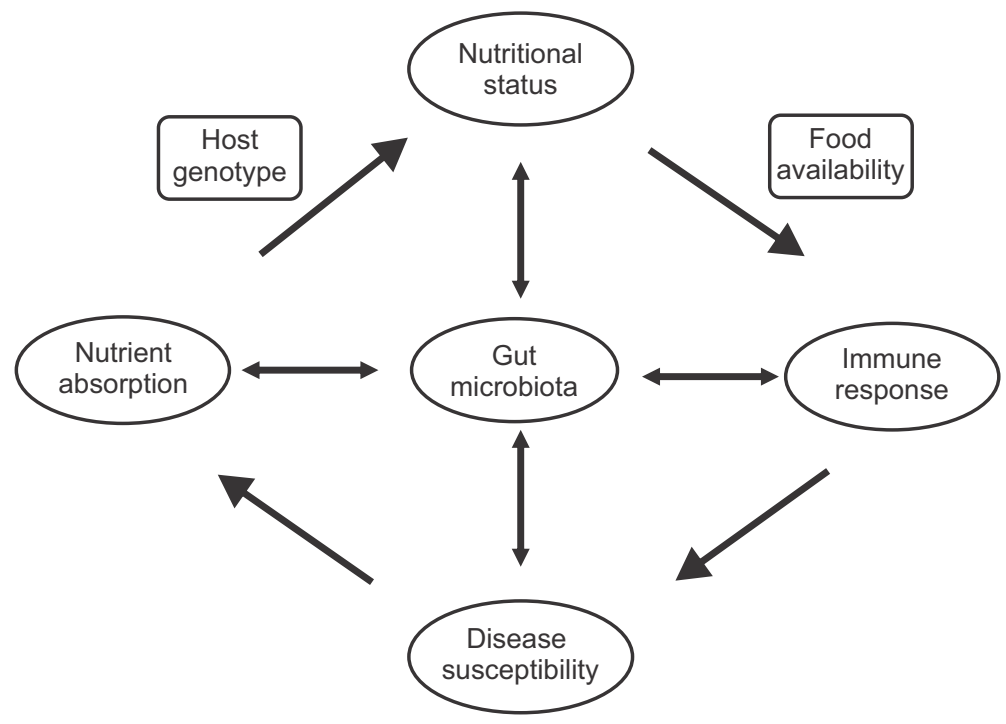

Fig 1: Proposed relationships between the gut microbiota, diet, diseases and dysbiosis 


\section{Probiotics}

The commensal microbiota is the subject of a diverse array of current and ongoing scientific study and investigations. Areas of study have involved disciplines ranging from microbiology, gastroenterology, and immunology to nutrition and food science. Modulating the microbiota thus, has the potential to improve the health of the intestinal tract as well as improve the immune system and enhance the bioavailability of nutrients. Modulating the microbiota may also reduce the risk of certain diseases. ${ }^{[34]}$ Research involving each of the above noted disciplines has focused on the possible role of probiotics in improving and enhancing the function of the microbiota. Although probiotics have been variously defined, the most commonly cited definition is that advanced by the Food and Agriculture Organization and the World Health Organization, as "live microorganisms which when administered in adequate amounts confer a health benefit on the host". ${ }^{[35]}$

Human gastrointestinal tract normally contains many different types of bacteria, yeast and viruses, collectively known as 'gut flora.' Certain illnesses or medications can knock off beneficial gut flora out of balance which causes all kinds of problems, and probiotics help restore it. The mechanisms by which probiotics may help achieve health benefits, involve modifying the composition or function of gut microbiota, improving host immune response, reducing colonic $\mathrm{pH}$, bolstering intestinal barrier function, stimulating cell development, inhibiting gut pathogens and fostering nutrient absorption. Thus there is significant interest within various research communities with respect to investigating the therapeutic, nutritional, and functional effects of probiotics on the microbiota, and ultimately on human health.

\section{Probiotics as investigational new drugs}

Use of probiotics for correcting dysbiosis involves treatment modalities to manipulate and restore the balance in the richness and diversity of intestinal microbiome. ${ }^{[3]}$ Probiotics may introduce beneficial functions into the gastrointestinal tract or enhance the functionality of existing microbial communities. Probiotics may affect the composition and function of existing microbial communities by competing for nutrients, production of growth substrates or inhibitors.
Probiotics may also modulate host intestinal immunity. ${ }^{[37]}$ This concept is supported by results from randomized controlled clinical trials showing the benefits of probiotics during the treatment of gastrointestinal diseases.

Probiotic ability is often strain dependent and it is impossible to distinguish strains by basic micro biological methods. Cell constituents such as peptidoglycans, lipoteichoic acids and DNA of Lactobacillus rhamnosus GG acted for immuno-potentiation of the immune system influencing brain function and behavior, resulting in relieving depression. ${ }^{[1]}$ Further probiotics produce antimicrobial agents or metabolic compounds that suppress the growth of other microorganisms ${ }^{[38,39]}$ or compete for receptors and binding sites with other intestinal microbes on the intestinal mucosa. ${ }^{[40]}$ Probiotic lactobacillus strains enhance the integrity of the intestinal barrier, which may result in maintenance of immune tolerance, decreased translocation of bacteria across the intestinal mucosa, and disease phenotypes such as gastrointestinal infections, irritable bowel syndrome (IBS) and IBD. ${ }^{[41]}$

Moreover, probiotics can modulate the intestinal immunity and alter the responsiveness of the intestinal epithelia and immune cells to microbes in the intestinal lumen. ${ }^{[42,43]}$ A clinical study demonstrated decreased pain and flatulence in patients with IBS that receiving a 4-week treatment with a rose-hip drink containing $5 \times 10^{7}$ colony-forming units / $\mathrm{ml}$ of L. plantarum DSM 9843 per day. ${ }^{[4]}$ This improvement in clinical symptoms was associated with the presence of L. plantarum in rectal biopsies of patients, along with reduced amounts of enterococci in fecal specimens. A more recent study focusing on patients with diarrhea-dominant IBS (IBS-D) yielded symptomatic relief in patients treated with a probiotic consortia of L. acidophilus, L. plantarum, L. rhamnosus, Bifidobacterium breve, B. lactis, B. longum and Streptococcus thermophilus. After probiotic treatment, microbial composition was more stable and similar to placebo group. ${ }^{[45]}$

Cox et $a l^{[46]}$ analyzed the fecal microbiota of 6-month old infants treated with daily supplements of $L$. rhamnosus (LGG) using a high-throughput, cultureindependent method. Results showed an abundance of LGG and an increased uniformity in the fecal microbiota of these infants, suggesting ecological stability. The 
ability of probiotics to induce changes in intestinal microbial communities was demonstrated by a recent study, which explored the effects of $L$. reuteri on microbial community composition in a neonatal mouse model using $16 \mathrm{~S}$ rRNA metagenomic sequencing. The results showed uniformity in composition of gut microbial communities during the period animals were fed L. reuteri as compared to control animals. However the diversity of the distal intestinal microbiome increased post treatment. ${ }^{[4]}$

Probiotics may induce changes in the intestinal microbiota and stabilize microbial communities. However, further studies in humans are needed to assess whether probiotics can make the same impact on the human intestinal microbiome and whether the changes are associated with clinical benefits in the host. In addition to directly affecting the composition of the intestinal microbiota, probiotics may also modulate the global metabolic function of intestinal microbiomes.

\section{Probiotics and regulation of IND}

An IND needs an authorization from Food and Drug Administration (FDA) similar to any drug molecule to be administered as an investigational drug or biological product to humans. Such authorization must be sought prior to interstate shipment and administration of any new drug or biological product that is not the subject of an approved New Drug Application or Biologics/ Product License Application. Probiotics that are intended to be used to diagnose, cure, mitigate, treat, or prevent disease in humans or other animals and that affect the structure or the function of the body are considered to be drugs. ${ }^{[4]}$ The development pathway for a "biologic new drug" is similar to that of any new drug. Following the discovery, nonclinical safety testing precedes clinical safety testing and then efficacy testing. This approach is based on the new drug provisions of the Food, Drug, and Cosmetic Act, which is further defined by federal regulation.

The IND application exempts an unapproved new drug from the requirements of a marketed drug (i.e., safety and efficacy). An IND application must be filed when a clinical investigation is to be conducted on an article when the article is an "unapproved" drug (i.e., not marketed as a drug in the United States) or when the article is an "approved" drug and the clinical investi- gation is intended to significantly "change labeling and/or current use, route, advertising, promotion, etc. "Clinical investigation" means any experiment in which a drug is administered to one or more human subjects. ${ }^{[9]}$ An "unapproved" drug is a product not generally recognized as safe and effective when used as labeled (also called a "new" drug), as defined in Section 201(p) of the Food, Drug, and Cosmetic Act (Section 321(p)) (standard language from sample FDA Warning Letters). Since there are currently no probiotics approved as "drugs" in the United States, a probiotic sponsor is required to file an IND application with the FDA before the initiation of clinical trials for a "drug" indication. The conditions for filing an IND application exclude the use of the product under the "practice of medicine." Practice of medicine is the use of any product legally available in the United States to a licensed health practitioner for the management of his or her patients, as long as the practitioner is not manufacturing, marketing, advertising, or promoting the product for an "unapproved" use, if premarketing approval for that indication is required. This scenario includes the "offlabel" use of approved drugs. ${ }^{[48]}$

\section{Commercialized probiotic products and supplements}

Probiotics exist naturally in foods such as yogurts, cultured milks i.e. buttermilk, kefir, etc., and fermented foods such as fresh sauerkraut, kimchi, tempeh, and miso. However recent era has witnessed enormous interest in commercialization of such beneficial probiotic strains for making them available to consumers. A list of various commercially proven probiotic strains has been developed by the California Dairy Research Foundation. Different product formulations of these clinically proven probiotic strains are available in the market which claims a variety of health benefits. One of such products with a very high consumer acceptability is "Yakult". It is a commercialized fermented milk drink containing over 6.5 billion cells of $L$. casei strain Shirota. It improves digestion, gut health and immunity in humans when consumed frequently. ${ }^{[51-53]}$

Fortification with probiotic bacteria lead to functional food preparations in the form of nutritional supplements that can ameliorate specific deficiencies like anemia, vitamin and mineral deficiencies, etc. Yogurt based products fortified with additional 
probiotics are being manufactured now-a-days for people of all ages. A variety of fermented milk based drinks fortified with specific probiotic strains are available in the market. Nutrifit fermented milk manufactured by Mother Dairy, (India) boosts immunity in humans when consumed every day. Actimel yoghurt drink by Dannon, contains 10 billion L. casei along with calcium and vitamins B6 and D and is proven to strengthen body's natural defenses. Iron and folic acid fortified yogurt is available for consumption by pregnant women. A creamy low fat yogurt named Activia is manufactured by Dannon. It contains probiotic Bifidobacterium lactis DN-173 010 which helps in regulation of human digestive system. This Yogurt has been made available in seven flavors to cater to a variety of consumer choices. Fiberone Nonfat Yogurt has been fortified with chicory root which gives each container of yogurt five grams of fiber. It is additionally fortified with calcium, vitamin $\mathrm{A}$ and $\mathrm{D}$ also. ${ }^{[54-57]}$

Probiotic dietary supplements like Culturelle ${ }^{\circledR}$ are available in the market. Culturelle ${ }^{\circledR}$ contains Lactobacillus GG along with trace amounts of dairy proteins. No wheat or gluten is present in it. It is helpful in promoting overall digestive and immune health. ${ }^{[58-60]}$ Further, a cereal based product named Vive which contains whole grains, calcium carbonate, natural flavor, soy protein concentrate, Lactobacillus probiotic cultures, (L. acidophilus or L. casei), vitamin D etc. has been manufactured by Kashi company in United States. This nutritious product enhances digestive wellness of body.

A lemon ginger probiotic tea along with the herbs contains GanedenBC probiotics is available. It has lemon flavor with the hint of ginger. It promotes healthy digestion. In Sweden, the Skane ${ }^{\circledR}$ company launched an extension of "ProViva ${ }^{\circledR}$ " probiotic fruit juice drink, based on clinical evidence that such microorganisms can improve iron intake in women. ${ }^{[49]}$ Various probiotic based capsule formulations are also available for women ${ }^{[50]}$ TravelProbio capsules which are a probiotic blend of Lactobacillus, acidophilus, B. lactis, L. bulgaricus fortified with a prebiotic fructooligosaccharides (FOS), help to build body's resistance to gut pathogens before and during travel.

Commercially available brand of NOW Probiotic Defense capsules are a blend of probiotics in a whole food base and contain 1 billion bacteria, including soil organisms. These promote positive probiotic balance and support healthy immune system. iFlora ${ }^{\circledR}$ MultiProbiotic ${ }^{\circledR}$ Capsules by Sedona Labs contain 32 billion active cells per serving of 16 probiotic strains along with non-gas forming ScFOS ${ }^{\circ}$ prebiotic fiber for optimum synbiotic support. The formulation contains various potent strains of Lactobacillus, Bifidobacterium, Streptococcus and a clinically researched strain B. lactis HN019 to support optimal large intestine health and promote a healthy immune system. ${ }^{[61-64]}$

\section{CONCLUSION}

Existence of metabolic disorders has a significant association with microbiome alterations occurring in the intestinal tract with progression of disease. Probiotic interventions are thought to contribute positively towards prevention and treatment of metabolic disorders that emerged due to microflora imbalance or dysbiosis in gut. Since preclinical and clinical trials are mandatory for practical biomedical applications, probiotics have been considered under category of INDs and INDs which must follow the approval procedure similar to drugs. Further investigation needs to be carried out for finding more probiotic strains with implications in treatment and prevention of metabolic disorders among humans and animals.

\section{SOURCE OF FUNDING}

Department of Science and Technology, Govt. of India (Project No. SR/SO/HS 38/2009)

\section{ACKNOWLEDGEMENTS}

The authors are thankful to the funding agency for providing financial assistance to TK under Major Research Project awarded to PPB.

\section{CONFLICT OF INTEREST : None declared}

\section{REFERENCES}

1. Herbel SR, Vahjen W, Wieler LH, Guenther S. Timely approaches to identify probiotic species of the genus Lactobacillus. Gut Pathog. 2013;5:27.

2. Palmer C, Bik EM, Digiulio DB, Relman DA, Brown PO. Development of the human infant intestinal microbiota. PLoS Biol. 2007;5:e177.

3. Zoetendal EG, Rajilic-Stojanovic M, de Vos WM. High-throughput diversity and functionality analysis of the gastrointestinal tract microbiota. Gut. 2008;57:1605-15.

4. O'Toole PW, Claesson MJ. Gut microbiota: Changes throughout 
the lifespan from infancy to elderly. Int Dairy J. 2010;20:281-91.

5. Dethlefsen L, Huse S, Sogin ML, Relman DA. The pervasive effects of an antibiotic on the human gut microbiota, as revealed by deep 16S rRNA sequencing. PLoS Biol. 2008;6:e280.

6. Raes J, Foerstner KU, Bork P. Get the most out of your metagenome: Computational analysis of environmental sequence data. Curr Opin Microbiol. 2007;10:490-8.

7. Manichanh C. Reduced diversity of faecal microbiota in Crohn's disease revealed by a metagenomic approach. Gut. 2006; 55:205-11.

8. Sokol H, Pigneur B, Watterlot L, Lakhdari O, Bermudez-Humaran LG, Gratadoux J-J, et al. Faecalibacterium prausnitzii is an antiinflammatory commensal bacterium identified by gut microbiota analysis of Crohn disease patients. Proc Natl Acad Sci USA. 2008;16731-6.

9. Koenig J, Spor A, Scalfone N, Fricker A, Stombaugh J, Knight R, et al. Succession of microbial consortia in the developing infant gut microbiome. Proc Natl Acad Sci USA. 2011;108:4578-85.

10. Emerson BC, Gillespie RG. Phylogenetic analysis of community assembly and structure over space and time. Trends Ecol Evol. 2008;23:619-30.

11. Cavender-Bares J, Kozak KH, Fine PV, Kembel SW. The merging of community ecology and phylogenetic biology. Ecol Lett. 2009;12:693-715.

12. Favier CF, Vaughan EE, De Vos WM, Akkermans AD. Molecular monitoring of succession of bacterial communities in human neonates. Appl Environ Microbiol. 2002;68:219-26.

13. Turnbaugh PJ, Ridaura VK, Faith JJ, Rey FE, Knight R, Gordon $\mathrm{Jl}$. The effect of diet on the human gut microbiome: a metagenomic analysis in humanized gnotobiotic mice. Sci Trans Med 2009;1:6ra14-6ra14. doi: 10.1126/scitransImed.3000322.

14. David LA, Maurice CF, Carmody RN, Gootenberg DB, Button JE, Wolfe $\mathrm{BE}$, et al. Diet rapidly and reproducibly alters the human gut microbiome. Nature. 2012;505:559-63.

15. Kau AL, Ahern PP, Griffin NW, Goodman AL, Gordon JI. Human nutrition, the gut microbiome and the immune system. Nature 2011;474:327-36.

16. Gupta SS, Mohammed MH, Ghosh TS, Kanungo S, Nair GB, Mande SS, et al. Metagenome of the gut of a malnourished child. Gut Pathog 2011;3:7.

17. Lawley TD, Walker AW. Intestinal colonization resistance. Immunol. 2013;138:1-11.

18. Morgan XC, Segata N, Huttenhower C. Biodiversity and functional genomics in the human microbiome. Trends Genet. 2012;29:51-58.

19. He M, Miyajima F, Roberts P, Ellison L, Pickard DJ, Martin MJ, et al. Emergence and global spread of epidemic healthcare associated Clostridium difficile. Nat Genet. 2013;45:109-13.

20. Clements ACA, Magalhes RJS, Tatem AJ, Paterson DL, Riley TV. Clostridium difficile PCR ribotype 027: assessing the risks of further worldwide spread. Lancet Infect Dis. 2010;10:395-404.

21. Sekirov I, Russell SL, Antunes LCM, Finlay BB. Gut microbiota in health and disease. Physiol Rev. 2010;90:859-904.

22. Borody $\mathrm{TJ}$, Khoruts $\mathrm{A}$. Fecal microbiota transplantation and emerging applications. Nat Rev Gastroenterol Hepatol. 2012;9:88-96

23. Qin J, Li R, Raes J, Arumugam M, Burgdorf KS, Manichanh C, et al. A human gut microbial gene catalogue established by metagenomic sequencing. Nature. 2010;464:59-65.

24. Fraher $\mathrm{MH}$, O'Toole PW, Quigley EMM. Techniques used to characterize the gut microbiota: a guide for the clinician. Nat Rev Gastroenterol Hepatol. 2012;9:312-22.

25. Dave M, Higgins PD, Middha S, Rioux KP. The human gut microbiome: current knowledge, challenges, and future directions. Transl Res. 2012;160:246-57.

26. Maurice CF, Haiser HJ, Turnbaugh PJ. Xenobiotics shape the physiology and gene expression of the active human gut microbiome. Cell. 2013;52:39-50.

27. Turnbaugh PJ, RE Ley, Mahowald MA, Magrini V, Mardis ER, Gordon Jl, et al. An obesity-associated gut microbiome with increased capacity for energy harvest. Nature. 2006;444:102731.

28. Turnbaugh PJ, Hamady M, Yatsunenko T, Cantarel BL, Duncan A, Ley RE, et al. A core gut microbiome in obese and lean twins. Nature. 2009;457:480-4.

29. Duncan SH, Lobley GE, Holtrop G, Ince J, Johnstone AM, Louis P, et al. Human colonic microbiota associated with diet, obesity and weight loss. Int J Obesity. 2008;32:1720-4.

30. Schwiertz A, Tara D, Schafer K, Beijer S, Bos NA, Donus C et al. Microbiota and SCFA in lean and overweight healthy subjects. Obesity. 2010;18:190-5.

31. Frank DN, St Amand AL, Feldman RA, Boedeker EC, Harpaz N, Pace NR. Molecular-phylogenetic characterization of microbia community imbalances in human inflammatory bowel diseases. Proc Natl Acad Sci USA. 2007;104:13780-5.

32. Kotlowski R, Bernstein CN, Sepehri S, Krause DO. High prevalence of Escherichia coli belonging to the $\mathrm{B} 2+\mathrm{D}$ phylogenetic group in inflammatory bowel disease. Gut. 2007; 56:669-75

33. Claesson MJ, Cusack S, O'Sullivan O, Greene-Diniz R, de Weerd $\mathrm{H}$, Flannery $\mathrm{E}$, et al. Composition, variability, and tempora stability of the intestinal microbiota of the elderly. PNAS. 2011;108:4586-91.

34. Collado MC. Role of Probiotics in Health and Diseases. In: Lee, K.L. \& Salminen, S. (eds) Handbook of Probiotics and Prebiotics. $2^{\text {nd }}$ edn. John Wiley and Sons, 2009.

35. Food and Agriculture Organization of the United States. Health and Nutritional Properties of Probiotics in Food Including Powdered Milk With Live Lactic Acid Bacteria. http://www.who. int/foodsafety/publications/fs_management/en/probiotics. pdf. 2001.

36. Sonnenburg J, Fischbach M. Community health care: therapeutic opportunities in the human microbiome. Sci Transl Med. 2011 3:78ps12. doi: 10.1126/scitranslmed.3001626

37. O'Toole $\mathrm{P}$, Cooney J. Probiotic bacteria influence the composition and function of the intestinal microbiota. Interdiscip Perspect Infect Dis. 2008;2008:175-85.

38. Spinler J, Taweechotipatr M, Rognerud C, Ou C, Tumwasorn S, Versalovic J. Human derived probiotic Lactobacillus reuter demonstrate antimicrobial activities targeting diverse enteric bacterial pathogens. Anaerobe. 2008;14:166-71.

39. O'Shea E, Cotter P, Stanton C, Ross R, Hill C. Production of bioactive substances by intestinal bacteria as a basis for explaining probiotic mechanisms: bacteriocins and conjugated linoleic acid. Int J Food Microbiol. 2012;152:189-205.

40. Collado M, Meriluoto J, Salminen S. Role of commercial probiotic strains against human pathogen adhesion to intestinal mucus. LettAppl Microbiol. 2007;45:454-60.

41. Lee B, Bak Y. Irritable bowel syndrome, gut microbiota and probiotics. J Neurogastroenterol Motil. 2011;17:252-66.

42. Thomas C, Hong T, Van Pijkeren J, Hemarajata P, Trin D, Hu W et al. Histamine derived from probiotic Lactobacillus reuteri suppresses TNF via modulation of Pka and Erk signaling. PLoS ONE. 2012;7:e31951. doi: 10.1371/journal.pone.0031951.

43. Bron $\mathrm{P}$, Van Baarlen $\mathrm{P}$, Kleerebezem M. Emerging molecular insights into the interaction between probiotics and the host intestinal mucosa. Nat Rev Microbiol. 2011;10:66-78. 
44. Nobaek S, Johansson M, Molin G, Ahrne S, Jeppsson B. Alteration of intestinal microflora is associated with reduction in abdominal bloating and pain in patients with irritable bowel syndrome. Am J Gastroenterol. 2000;95:1231-8.

45. Ki Cha B, Mun Jung S, Hwan Choi C, Song I, Woong Lee H, Joon $\mathrm{Kim} \mathrm{H}$, et al. The effect of a multispecies probiotic mixture on the symptoms and fecal microbiota in diarrhea-dominant irritable bowel syndrome: a randomized, double-blind, placebo-controlled trial. J Clin Gastroenterol. 2011;46:220-7.

46. Cox M, Huang Y, Fujimura K, Liu J, McKean M, Boushey H. et al. Lactobacillus casei abundance is associated with profound shifts in the infant gut microbiome. PLoS One 2010;5: e8745. doi: 10.1371/journal.pone.0008745

47. Preidis G, Saulnier D, Blutt S, Mistretta T, Riehle K, Major A. et al. Probiotics stimulate enterocyte migration and microbial diversity in the neonatal mouse intestine. FASEB J. 2012;26: 1960-9.

48. Hoffman FA. Development of Probiotics as Biologic Drugs. Clin Infect Dis. 2008;46:S125-S127.

49. Lepay DA. Emerging issues in FDA's oversight of clinical research. Presented at the Food and Drug Administration Science Board Meeting, 16 November 2001.

50. List developed by the California Dairy Research Foundation (January 2012) available at http://cdrf.org/home/checkoffinvestments/usprobiotics/products-with probiotics/\# commercial http://cdrf.org/home/checkoff-investments/usprobiotics/ products-with-probiotics/\#commercial.

51. Nagao F, Nakayama M, Muto T, Okumura K. Effects of a fermented milk drink containing Lactobacillus casei strain Shirota on the immune system in healthy human subjects. Biosci Biotechnol Biochem. 2000;64:2706-8.

52. Sgouras D, Maragkoudakis P, Petraki K, Martinez-Gonzalez B, Eriotou E, Michopoulos S, Mentis A. In vitro and in vivo inhibition of Helicobacter pylori by Lactobacillus casei strain Shirota. Appl Environ Microbiol. 2004;70:518-26.

53. Lee JW, Kim EH, Yim IB, Joo HG. Immunomodulatory and antitumor effects in vivo by the cytoplasmic fraction of Lactobacillus casei and Bifidobacterium longum. J Vet Sci. 2004; 5:41-8.

54. Bouvier M, Meance S, Bouley C, Berta JL, Grimaud JC. Effects of consumption of a milk fermented by the probiotic Bifidobacterium animalis DN-173 010 on colonic transit time in healthy humans. Biosci Microflora. 2001;20:43-8.
55. Marteau P, Cuillerier E, Meance S, Gerhardt MF, Myara A Bouvier M, et al. Bifidobacterium animalis, strain DN-173 010 shortens the colonic transit time in healthy women. A double-blind randomised controlled study. Aliment Pharmacol Ther. 2002;16:587-93.

56. Nishida $\mathrm{S}$, Ishikawa $\mathrm{Y}$, lino $\mathrm{H}$. Effect of Bifidobacterium lactis DN173010 on the Intestinal Transit Time, the Condition of Defecation and Intestinal Microflora: A Randomized, Double-blind, Placebocontrolled, Cross-over Study among Healthy Japanese Women. Pharmacometrics. 2008;74:99-106.

57. Guyonnet D, Schlumberger A, Mhamdi L, Jakob S, Chassany O. Fermented milk containing Bifidobacterium lactis DN-173 010 improves gastrointestinal well-being and digestive symptoms in women reporting minor digestive symptoms: a randomized, double-blind, parallel, controlled study. Br J Nut. 2009;102:165462.

58. Doron S, Snydman DR, Gorbach SL. Lactobacillus GG: bacteriology and clinical applications. Gastroenterol Clin North Am. 2005;34:483-98.

59. Kekkonen RA, Lummela N, Karjalainen H, Latvala S, Tynkkynen $\mathrm{S}$, Jarvenpaa $\mathrm{S}$ et al. Probiotic intervention has strain-specific anti-inflammatory effects in healthy adults., World $\mathrm{J}$ Gastroenterol. 2008;14:2029-36.

60. Lebeer S, Claesa I, Tytgata HLP, Verhoevena TLA, Mariena E von Ossowski I et al. Functional analysis of the pili of Lactobacillus rhamnosus GG in relation to adhesion and immunomodulatory interactions with intestinal epithelial cells. Appl Environ Microbiol. 2012;78:185-93.

61. Arunachalam K, Gill HS, Chandra RK. Enhancement of natural immune function by dietary consumption of Bifidobacterium lactis (HN019). Eur J Clin Nutr. 2000;54:263-7.

62. Gill HS, Rutherfurd KJ, Cross ML. Dietary probiotic supplementation enhances natural killer cell activity in the elderly: an investigation of age-related immunological changes. J Clin Immunology. 2001;21:264-71.

63. Ahmed M, Prasad J, Gill H, Stevenson L, Gopal P. Impact of consumption of different levels of Bifidobacterium lactis HN019 on the intestinal microflora of elderly human subjects. J Nutr Health Aging. 2007;11:26-31.

64. Waller PA, Gopal PK, Leyer GJ, Ouwehand AC, Reifer C, Stewart ME, Miller LE. Dose-response effect of Bifidobacterium lactis HN019 on whole gut transit time and functional gastrointestinal symptoms in adults. Scand J Gastro. 2011:46:1057-64 